SRC TR $85-50$

Decomposition and Stability of Multiparameter Singular

Perturbation Problems

by

E. H. Abed 


\title{
Decomposition and Stability of Multiparameter Singular Perturbation Problems
}

\author{
Eyad H. Abed * \\ Department of Electrical Engineering \\ and Systems Research Center \\ University of Maryland, College Park, MD 20742
}

\begin{abstract}
Time-scale separation and stability of linear time-varying and time-invariant multiparameter singular perturbation problems are analyzed. The first problem considered in the paper is that of deriving upper bounds on the small 'parasitic' parameters ensuring the existence of an invertible, bounded transformation exactly separating fast and slow dynamics. This problem is most interesting for the time-varying case. The analysis of this problem in the time-varying case requires the two time-scale setting introduced by H.K. Khalil and P.V. Kokotovic (SIAM J. Control Optim., 17, 56-65, 1979). This entails that the mutual ratios of the small parameters are bounded by known positive constants. The second problem considered is to derive parameter bounds ensuring that the system in question is uniformly asymptotically stable. The results on decomposition are used to facilitate the derivation of these latter bounds. Fortunately, the analysis of decomposition and stability questions for time-invariant multiparameter singular perturbation problems requires no restriction on the relative magnitudes of the small parameters. A concept of 'strong D-stability' is introduced and shown to greatly simplify the stability analysis of tirne-invariant multiparameter problems.
\end{abstract}

Revised: December 1985

\footnotetext{
* Supported in paro by the NSF under Grants ECS-84-04275 and OIR-85-00108, by a grant from the Minta Martin Fund for Aeronautical Research, Univ. of Maryland, and by the Power Engineering Program, Univ. of Maryland, through grants from BG\&E, PEPCO, VEPCO, Bechtel Corp. and the Creneral Electric Foundation.
} 


\section{INTRODUCTION}

Singular perturbation problems involving several small parameters have in the past attracted considerable attention, both from the engineering and the applied mathematics communities. This is due in part to the difficulty of these problems and in part to their wide applicability in power system dynamics, control of large scale systems, multi-modeling, differential games, and similar settings. The small singular perturbation parameters typically represent unmodeled 'parasitic' capacitances, inductances, time constants, inertia constants, inverses of high feedback gains, etc. It has long been recognized that the analysis of many problems in singular perturbations is considerably simplifled if a decomposition of 'fast' and 'slow' dynamics can be achieved. Such a decomposition of time scales is realized through a transformation of variables whose existence must of course be proved.

This paper has three main goals. The flrst is to derive explicit upper bounds on the singular perturbation parameters ensuring the existence of an invertible, bounded transformation decoupling fast and slow dynamics. To the author's knowledge, this type of result has not previously been reported for the case of time-varying singularly perturbed systems (with one small parameter or several). Second, the decoupled equations are employed in the derivation of further upper bounds on the small parameters guaranteeing asymptotic stability. The third goal is to obtain unified stability results for general multiparameter singular perturbation problems which apply regardless of the relative magnitudes of the small parameters. The paper achieves this last goal for the case of time-invariant multiparameter singular perturbation problems, though in the general time-varying case this remains an open problem.

Linear dynamical equations of the form

$$
\begin{aligned}
\dot{x} & =A(t) x+B(t) y \\
\epsilon_{i} \dot{y}_{i} & =C_{i}(t) x+D_{i}(t) y, \quad i=1, \ldots, M .
\end{aligned}
$$

are the subject of this work. The case in which (1.1) is a time-invariant equation will be considered separately from the general time-varying case. In Eq. (1.1), $x \in R^{n}$, $y=\left(y_{1}, \ldots, y_{M}\right) \in R^{m}, \quad y_{i} \in R^{m_{\imath}}, \epsilon=\left(\epsilon_{1}, \ldots, \epsilon_{M}\right)$ with each $\epsilon_{i}$ a small real parameter, $A, B, C_{i}, D_{i}$ are real matrices of conformable dimensions, and the dot signifles differentiation with respect to time $t$. The asymptotic analysis of (1.1) in the limit $\epsilon_{i} \rightarrow 0, i=1, \ldots, M$ is an example of a singular perturbation problem. This means that the order of the system (1.1) differs for $\epsilon_{i}=0$ and $\epsilon_{i} \neq 0$. For a discussion of control-theoretic results in singular perturbation theory, see the excellent review articles $[1]-[3]$.

Parameter bounds ensuring the existence of a decoupling transformation for time-invariant problems with a single small parameter have previously been reported in [4]-[7]. Considerably greater effort has been devoted to obtaining parameter bounds ensuring the stability of singularly perturbed systems [6]-\{15]. The upper bound of Khalil [11] is of the latter type, and is derived for (nonlinear) time-invariant multiparameter problems (cf. also Khorasani and Pai [15]). This paper also gives parameter bounds for stable time-invariant multiparameter singular perturbation problems which do not suffer from a certain conservativeness problem which is present in [11], and which occurs also in the results of this paper for the time-varying case.

Most previous studies of singularly perturbed systems containing several small parameters begin by hypothesizing some type of relationship among the small parameters $\epsilon_{i}$. The simplest of these results in a standard single parameter singular perturbation problem. This is achieved by assuming that the parameters $\epsilon_{i}$ are known multiples of a single, auxiliary small parameter $\delta$, so that $\epsilon_{i}=\alpha_{i} \delta, i=1, \ldots, M$. Khalil and Kokotovic have pointed out in [16] that this assumption is not justifiable in many cases of practical interest, if only because of the lack of knowledge of the coefficients $\alpha_{i}$. As a more realistic assumption, [16] allows the parameters $\epsilon_{i}$ to be arbitrary subject to the requirement that the mutual ratios $\epsilon_{i} / \epsilon_{j}, i, j=1, \ldots, M$ are bounded by known positive constants. This is equivalent to constraining $\epsilon$ to lie in a (linear) cone in the positive orthant $R_{\Psi}^{M}$. The authors of [16] refer to this as the "multiparameter assumption." This assumption is also invoked in $[11],[15 j,[17 j-\{\geq 0]$ and, for tirne-varying systems, in the present paper. A perhaps 
more common hypothesis is that of multiple time scales [20]-[26]. This requires that the small parameters $\epsilon_{i}$ are of different orders of magnitude, say $\epsilon_{i+1} / \epsilon_{i} \rightarrow 0$ as $\epsilon_{i} \rightarrow 0$. The multiparameter setting of [16] clearly differs drastically from this case; indeed only two time scales are present in the setting of [16], just as for single parameter singular perturbation problems.

Although the results on time-varying systems in this paper require Khalil and Kokotovic's assumption that the mutual ratios $\epsilon_{i} / \epsilon_{j}$ are bounded, the results on time-invariant systems apply without any such restriction. The latter results yield each of the foregoing multiparameter singular perturbation hypotheses as special cases. Similar results have previously been obtained by the author in [27]-[30], which contain various stability and bifurcation results for time-invariant multiparameter problems without limiting a priori the way in which $\epsilon \rightarrow 0$. This author is of the opinion that use of the term multiparameter singular perturbation should be expanded to include all possible assumptions on the manner in which the small parameters $\epsilon_{i}$ are constrained, including even the multiple time-scale setting discussed above.

The development of the paper is as follows. Section II introduces the problem and provides relevant background material. Several hypotheses used at various stages in the development are also given in Section II. Parameter bounds for decomposition and stability of time-varying multiparameter problems are derived in Section IIr. Section III.1 is devoted to a constructive proof of the existence of a decoupling transformation which separates the fast and slow dynamics of (1.1). Two upper bounds on the small parameters are obtained there. These ensure the existence of a bounded decoupling transformation as well as the uniform asymptotic stability of an associated fast subsystem. In Section III.2 a further upper bound is obtained ensuring the uniform asymptotic stability of the slow subsystem. These three upper bounds are collected to yield an upper bound ensuring uniform asymptotic stability of the original multiparameter singularly perturbed system (1.1). This appears in Section III.3. Section III.4 contains an example illustrating the computation of the parameter bounds for the time-varying case. Section IV develops results parallel to those of Section III for time-invariant multiparameter singular perturbation problems. Conclusions and some open questions are given in Section $\mathrm{V}$.

Notation. Throughout the paper the Euclidean norm is used for vectors and the Frobenius norm for matrices. This is only for simplicity of the development, and does not represent a limitation of the method. The Euclidean and Frobenius norms are compatible. Recall that the Frobenius norm of a real matrix $A$ is the square root of the sum of the squares of all the elements of $A$. It will be denoted by $|A|$. If $A$ depends continuously on time $t$ and is bounded, its norm is $\|A\|$ $:=\sup _{t \geq 0}|A(t)|$. With the hope that there will be no confusion, this is denoted simply by $|A|$. The transpose of $A$ is indicated by $A^{\prime}$. If $A$ is a square matrix, $\sigma(A)$ denotes the spectrum or set of eigenvalues of $A$.

\section{PRELIMINARY CONSIDERATIONS}

It is useful to express Eq. (1.1) in the more compact form

$$
\begin{aligned}
\dot{x} & =A(t) x+B(t) y \\
E(t) \dot{y} & =C(t) x+D(t) y .
\end{aligned}
$$

Here $C(t):=$ block col $\left(C_{1}(t), \ldots, C_{M}(t)\right), D(t):=$ block col $\left(D_{1}(t), \ldots, D_{M}(t)\right)$, and $E(\epsilon):=$ block diag $\left(\epsilon_{1} I_{m_{1}}, \ldots, \epsilon_{M} I_{m_{M}}\right)$, where $I_{k}$ denotes the $k \times k$ identity matrix. With (2.1) one associates the reduced system

$$
\begin{aligned}
& \dot{x}=A(t) x+B(t) y \\
& 0=C(t) x+D(t) y
\end{aligned}
$$

obtained by formally substituting $\epsilon=0$ in (2.1).

The following assumptions are now made about the matrices $A, B, C, D$. 
(H1) The matrices $A(t), B(t), C(t), D(t)$ are bounded and depend continuously on $t$ for $t \geq 0$.

(H2) There is a $d>0$ such that the eigenvalues of $D(t)$ all have magnitude $\geq d$ for all $t \geq 0$.

Hypothesis (H2) implies (2.2) is equivalent to the system

$$
\dot{x}=\left[A(t)-B(t) D^{-1}(t) C(t)\right] x=: A_{0}(t) x \text {. }
$$

It also implies that $D^{-1}(t)$ (and hence also $A_{0}(t)$ ) is bounded on $0 \leq t<\infty$. To see this, note that (H2) implies $|\operatorname{det} D(t)| \geq d^{m}$, so that Lemma 1 of Coppel [31, p. 47] implies

$$
\left|D^{-1}(t)\right| \leq\left(2^{m}-1\right) d^{-m}|D(t)|^{m-1}
$$

and the conclusion follows from (H1).

Recall $[32$, p. 276] that a matrix $F$ is said to be $D$-stable if the eigenvalues of $D F$ have strictly negative real parts for any diagonal matrix $D$ with strictly positive diagonal elements. The following generalization is due essentially to Khalil and Kokotovic [16].

Definition 1. The matrix $D(t)$ is said to be block $D$-stable relative to the multi-index $\left(m_{1}, \cdots, m_{M}\right)$ if for all $\theta_{i}>0, i=1, \ldots, M$,

$$
\operatorname{Re} \sigma(\Theta(\theta) D(t))<0
$$

for all $t \geq 0$, where $\Theta(\theta):=\operatorname{block} \operatorname{diag}\left(\theta_{1} I_{m_{1}}, \ldots, \theta_{M} I_{m_{M}}\right)$.

If $D(t)$ is not block D-stable, it may still be possible to find a set $I \subset R_{+}^{M}$ to which the next deflnition applies. The relevance of this deflnition was a main hypothesis in [16].

Definition 2. The matrix $D(t)$ possesses Property $D$ relative to the set $H \subset R_{+}^{M}$ if there is a $\sigma_{1}>0$ such that

$$
\operatorname{Re} \sigma\left(|\epsilon| E^{-1}(\epsilon) D(t)\right)<-\sigma_{1}
$$

for all $t \geq 0, \epsilon \in H$.

Remark 1. It is easy to verify that $D(t)$ cannot possess Property $\mathrm{D}$ relative to the positive orthant $R_{+}^{M}$, even if $D(t)$ is block D-stable according to Definition 1 . Property $\mathrm{D}$ can only hold on subsets of $R_{+}^{M}$ for which $|\epsilon| E^{-1}(\epsilon)$ is bounded, i.e. when the ratios $\epsilon_{i} / \epsilon_{j}$ are bounded.

The results on time-varying systems in this paper will apply as $\epsilon \rightarrow 0$ in any subset $H$ of $R_{+}^{M}$ for which all the mutual ratios $\epsilon_{i} / \epsilon_{j}$ are bounded. These sets are cones of the form specified by Eq. (2.11) of hypothesis (H4) below. This generality is achieved based on hypothesis (H3) below, which was discussed but not enforced in [16].

(H3) There exists a continuously differentiable block diagonal positive definite matrix $P(t)$ with $\dot{P}^{\circ}(t)$ bounded,

$$
P(t)==\text { block } \operatorname{diag}\left[P_{1}(t), \ldots, P_{M}(t)\right]
$$

satisfying

$$
c_{1}|y|^{p} \leq y^{\prime} P(t) y \leq c_{2}|y|^{2}, \text { for all } y \in R^{m}, t \geq 0,
$$

such that $Q(t)$ given by

$$
P(t) D(t)+D^{\prime}(t) P(t)=-Q(t)
$$

is positive definite, and moreover satisfies 


$$
y^{\prime} Q(t) y \geq c_{3}|y|^{2}, \text { for all } y \in R^{m}, t \geq 0 \text {. }
$$

In (2.7), $P_{i}(t) \in R^{m_{i}}, i=1, \ldots, M$.

This hypothesis implies that $D(t)$ is a block D-stable matrix (cf. Johnson [33], Khalil and Kokotovic [16] ). It has been noted to yield an interesting class of D-stable matrices [33]. It has also been employed by Khalil [11] to derive upper bounds on the small parameters for asymptotic stability of a class of nonlinear autonomous multiparameter singularly perturbed systems. Hypothesis (H3) is useful since it implies that $v(t, y)=y^{\prime} P(t) E(\epsilon) y$ is a Liapunov function for an associated boundary layer system (Eq. (2.12) below). The next hypothesis has been introduced in $[16]$.

(H4) The parameters $\epsilon_{i}$ have bounded mutual ratios. That is, there exist positive numbers $k_{i j}, K_{i j}, i, j=1, \ldots, M$ such that $\epsilon \in H$ where the cone $H \subset R^{M}$ is given by

$$
H:=\left\{\epsilon \in R_{+}^{M}: k_{i j} \leq \frac{\epsilon_{i}}{\epsilon_{j}} \leq K_{i j}\right\}
$$

It will become apparent in the sequel that a natural boundary layer system associated with (1.1) is

$$
\frac{d y}{d t}=E^{-1}(\epsilon) D(t) y
$$

Note that, contrary to the situation in single parameter singular perturbations, the boundary layer system depends on $\epsilon$. This is the essence of the difficulties encountered in multiparameter perturbations. One can also define the boundary layer system in a suitable sped-up time scale, such as $\tau:=t /|\epsilon|$.

Motivated by the single parameter theory, it is natural to seek conditions under which the behavior of (1.1) can be linked with that of the reduced system (2.3) and the boundary layer system (2.12). Results of this character (though not necessarily pertaining to stability) are the main goal of Khalil and Kokotovic [16] and of this paper. The proof of this paper results, for instance, in a computable upper bound $E_{0}$ on $|E(\epsilon)|=\left(m_{1} \epsilon_{1}{ }^{2}+\cdots+m_{M} \epsilon_{M}{ }^{2}\right)^{1 / 2}$ ensuring uniform asymptotic stability of (1.1). This is of course equivalent to obtaining an upper bound on a weighted norm of $\epsilon$. An (in general more conservative) upper bound on $|\epsilon|$ is easily obtained from these results (for any norm). An examination of the proof of [16] shows that it does not yield such an upper bound. This is mainly because [16] employs certain results of Coppel [34] which are based on compactness arguments. Note, however, that the results of [16] apply in case hypothesis (FI3) above is not in force, if $D(t)$ possesses Property $\mathrm{D}$ (cf. Definition 2 above) relative to a conic set $H$ of the form specified by Eq. (2.11). Hypothesis (H3) implies Property D, but the reverse implication does not hold [16]. 


\section{TIME-VARYING PROBLEMS}

\section{III.1. Decoupling of Fast and Slow Dynamics}

It is well known [1]-[4], [35] that for (nondegenerate) single parameter singularly perturbed systems it is possible to exhibit a nonsingular similarity transformation which exactly separates fast and slow dynamics. This transformation was presented by Chang [35] in the context of a general linear singularly perturbed boundary value problem. Chang's transformation is best understood as the composition of two simpler transformations. The flrst, derived by Chang in [36], results in block-triangularization of the system dynamics. The second transformation applied to the block-triangular system produces the desired block-diagonal (i.e., separated) form. A direct generalization of Chang's transformation to the multiparameter setting was applied to the stability analysis of multiparameter singularly perturbed systems by Khalil and Kokotovic [16]. A related transformation was used by this author to study multiparameter singularly perturbed Hopf bifurcation in [28] as well as to obtain general results on stability of time-invariant multiparameter singularly perturbed systems in $[27,29,30]$. In $[27,29,30]$ it was necessary to employ only the first step of Chang's transformation, yielding a block-triangular system. Also, the results of $[27,29,30]$ apply regardless of the relative magnitudes of the small parameters. In [16] the full transformation was employed to completely separate the fast and slow dy namics of a linear time-varying multiparameter singularly perturbed system of the form (1.1). This is necessary because of the time-varying nature of (1.1). Thus a complete separation of fast and slow dynamics will also be used in the present section, which is concerned with time-varying multiparameter singular perturbation problems. For an example illustrating the possible adverse effect of a (small) off-diagonal term on the stability of an otherwise stable linear time-varying system, see [37, pp. 151-153].

The results of Chang [35] (cf. also $[16,36]$ ) imply that the transformation

$$
\left(\begin{array}{l}
\eta \\
\xi
\end{array}\right)=\left(\begin{array}{ccc}
I & -M E(\epsilon) L & -M E(\epsilon) \\
L & I
\end{array}\right)\left(\begin{array}{l}
x \\
y
\end{array}\right)
$$

applied to (1.1) will result in the (decoupled) system

$$
\begin{aligned}
\dot{\eta} & =[A(t)-B(t) L(t, \epsilon)] \eta \\
E(\epsilon) \dot{\xi} & =[D(t)+E(\epsilon) L(t, \epsilon) B(t)] \xi
\end{aligned}
$$

provided $L(t, \epsilon)$ and $M(t, \epsilon)$ are solutions of the respective matrix differential equations

$$
\begin{aligned}
& E(\epsilon) \dot{L}=D L-C-E(\epsilon) L A+E(\epsilon) L B L \\
& \dot{M} E(\epsilon)=(A-B L) M E(\epsilon)-M(D+E(\epsilon) L B)+B
\end{aligned}
$$

defined for $0 \leq t<\infty$.

The transformation (3.1) is easily verifled to be a nonsingular similarity transformation for any value of $\epsilon$, for any matrices $L$ and $M$. See Eq. (3.47) of Section III.2 for the inverse transformation. Conditions will now be given for the equations (3.3) and (3.4) to have uniformly bounded solutions for all $\epsilon \in H$ (cf. (H4)) with $|\epsilon|$ sufficiently small.

Theorems 1 and 2 below show that under (H1)-(H4), uniformly bounded solutions of (3.3) and (3.4) exist on $0 \leq t<\infty$ for all $\epsilon \in H$ with $|\epsilon|$ sufficiently small. This fact follows from Lemmas 1 and 2 of $[16]$. Invoking hypothesis (H3), however, facilitates the constructive proofs presented below which, in addition, yield explicit upper bounds on $|E(\epsilon)|$ ensuring the existence of these bounded solutions.

Theorem 1. Under hypotheses (H1)-(H/), there is a scalar $E_{1}>0$ such that Eq. (3.3) has a solution $L(t, \epsilon)$ which is uniformly bounded for $|E(\epsilon)|<E_{1}, \epsilon \in H, t \geq 0$. Moreover, the solution with initial condition $L(0, \epsilon)=-D^{-1}(0) C(0)$ is uniformly bounded for $|E(\epsilon)|<E_{1}, \epsilon \in H$. 
Theorem 2. Let hypotheses (H1)-(H4) hold, and suppose there exists a uniformly bounded solution $L(t, \epsilon)$ of (3.3). Then there is a scalar $E_{2}>0$ such that Eq. (3.4) has a solution $M(t, \epsilon)$ which is uniformly bounded for $|E(\epsilon)|<E_{2}, \epsilon \in H, t \geq 0$. Moreover, $E_{2}$ may be chosen so that $|E(\epsilon)|<E_{2}$ also implies the uniform asymptotic stability of the null solution of the fast subsystem $(3.2 \mathrm{~b})$.

The proof of Theorem 1 will make use of the following elementary stability result, which is Lemma 1 in LaSalle and Lefschetz [38, pp. 116-117]. First some notation. Given a closed set $M \subset R^{n}$ and a positive scalar $r$, let $M_{r}$ denote the set of all points whose distance from $M$ is less than $r$. Also, let $M^{c}$, respectively $M_{r}^{c}$ denote the set of points outside $M$ (i.e., the complement of $M$ ), respectively $M_{r}$.

Proposition 1. Consider a system $\dot{x}=f(t, x), t \geq 0, x \in R^{n}$. Let $v(t, x)$ be a scalar function continuously differentiable in $t$ and $x$ for $t \geq 0, x \in R^{n}$, and let $M$ be a closed set in $R^{n}$. If $\dot{v}(t, x) \leq 0$ for all $x \in M^{c}$ and if $v\left(t_{1}, x_{1}\right)<\bar{v}\left(t_{2}, x_{2}\right)$ for all $t_{2} \geq t_{1} \geq 0$, all $x_{1} \in M$ and all $x_{2} \in M_{r}^{c}$, then each solution of $\dot{x}=f(t, x)$ which at some time $t_{0}$ is in $\bar{M}$ can never thereafter leave $M_{r}$.

Remark 2. From the proof of Proposition 1 in [38] it is clear that the conclusion of Proposition 1 holds if $\dot{v}(t, x) \leq 0$ is assumed to hold on $M_{r}-M$ rather than on all of $M^{c}$.

Proof of Theorem 1: It is straightforward to verify that the matrix differential equation (3.3) is equivalent to the vector differential equation

$$
\bar{E}(\epsilon) \dot{\lambda}(t)=\bar{D}(t) \lambda(t)-\bar{C}(t)-\bar{E}(\epsilon) \bar{L}(t) \bar{A}(t)+\bar{E}(\epsilon) \bar{L}(t) \bar{B}(t) \lambda(t),
$$

where the vector $\lambda \in R^{m n}$ is obtained from the $m \times n$ matrix $L=\left(L^{1}, \ldots, L^{n}\right)$ by concatenating the columns $L^{i}, i=1, \ldots, n$ of $L: \lambda^{\prime}:=\left(\left(L^{1}\right)^{\prime}, \ldots,\left(L^{n}\right)^{\prime}\right)$. Denote the columns of $A(t)$, respectively $C(t)$, by $A^{1}(t), \ldots, A^{n}(t)$, respectively $C^{1}(t), \ldots, C^{n}(t)$. The matrices $\bar{A}, \bar{B}, \bar{C}, \bar{D}, \bar{E}, \bar{L}$ appearing in Eq. (3.5) are defined as follows (here, block $\operatorname{diag}(X, \ldots, X)$ implies $n$ occurrences of $X$ in the parentheses, for any matrix $X$ ):

$$
\begin{aligned}
& \bar{A}(t):=\text { block col }\left(A^{1}(t), \ldots, A^{n}(t)\right) \in R^{n^{2}}, \\
& \bar{B}(t):=\text { block diag }(B(t), \ldots, B(t)) \in R^{n^{2} \times m n}, \\
& \bar{C}(t):=\text { block col }\left(C^{1}(t), \ldots, C^{n}(t)\right) \in R^{m n}, \\
& \bar{D}(t):=\text { block diag }(D(t), \ldots, D(t)) \in R^{m n \times m n}, \\
& \bar{E}(\epsilon):=\text { block diag }(E(\epsilon), \ldots, E(\epsilon)) \in R^{m n \times m n}, \\
& \bar{L}(t):=\text { block diag }(L(t), \ldots, L(t)) \in R^{m n \times n^{2}} .
\end{aligned}
$$

Note that $\bar{L}(t)$ in (3.5) depends linearly on $\lambda(t)$, by (3.6f) and the definition of $\lambda$.

Define the Liapunov function candidate $v(t, \lambda)$ by

$$
v(t, \lambda):=\lambda^{\prime} \bar{P}(t) \bar{E}(\epsilon) \lambda
$$

where $\bar{P}(t):=$ block diag $(P(t) \ldots, P(t))$. By (H3) $P(t)$ is block diagonal with the same structure as $E(\epsilon)$. Therefore $P(t)$ and $E(\epsilon)$ commute, implying that $\bar{P}(t)$ and $\bar{E}(\epsilon)$ also commute. 
Using this fact, $\dot{v}(t, \lambda)$ may be computed along trajectories of $(3.5)$ as

$$
\begin{aligned}
\dot{v}(t, \lambda)= & \lambda^{\prime}\left\{\bar{D}^{\prime}(t) \bar{P}(t)+\bar{P}(t) \bar{D}(t)\right\} \lambda+\lambda^{\prime}\left\{\frac{d}{d t} \bar{P}(t) \bar{E}(\epsilon)\right\} \lambda \\
& +\lambda^{\prime}\left\{\bar{B}^{\prime}(t) \bar{L}^{\prime}(t) \bar{P}(t) \bar{E}(\epsilon)+\bar{P}(t) \bar{E}(\epsilon) \bar{L}(t) \bar{B}(t)\right\} \lambda \\
& -\left\{\bar{C}^{\prime} \bar{P}(t) \lambda+\lambda^{\prime} \bar{P}(t) \bar{C}(t)\right\} \\
& -\left\{\bar{A}^{\prime}(t) \bar{L}^{\prime}(t) \bar{P}(t) \bar{E}(\epsilon) \lambda+\lambda^{\prime} \bar{P}(t) \bar{E}(\epsilon) \bar{L}(t) \bar{A}(t)\right\} .
\end{aligned}
$$

Now using Eqs. (2.9), (2.10) and the fact that $|\widetilde{L}|=n^{1 / 2}|\lambda|=\left|\bar{L}^{\prime}\right|$ (since $|\bar{L}|$ is the Frobenius norm of $\bar{L}),(3.8)$ implies

$$
\begin{aligned}
\dot{v}(t, \lambda) \leq & -c_{3}|\lambda|^{2}+|\bar{E}(\epsilon)|\left|\frac{d}{d t} \bar{P}\right||\lambda|^{2}+2 n^{1 / 2}|\bar{E}(\epsilon)||\bar{B}||\bar{P}||\lambda|^{\beta} \\
& +2|\bar{P}||\bar{C}||\lambda|+2 n^{1 / 2}|\bar{E}(\epsilon)||\bar{A}||\bar{P}||\lambda|^{2}
\end{aligned}
$$

Define $\mu:=|\bar{E}(\epsilon)|$ and the parametrized cubic polynomial $p_{\mu}(\alpha)$ by

$$
\begin{aligned}
p_{\mu}(\alpha):=\{ & \left.2 \mu n^{1 / 2}|\bar{B}||\bar{P}|\right\} \alpha^{3}+\left\{\mu\left|\frac{d}{d t} \bar{P}\right|+2 \mu n^{1 / 2}|\bar{A}||\bar{P}|-c_{3}\right\} \alpha^{2} \\
& +\{2|\bar{P}||\bar{C}|\} \alpha .
\end{aligned}
$$

Note that for $\mu=0, p_{\mu}(\alpha)$ reduces to a quadratic which takes negative values for all $\alpha>\left(2|\bar{P}||\bar{C}| / c_{3}\right)$. For small $\mu>0$ the cubic term dominates for large $\alpha$ and $p_{\mu}(\alpha)$ is positive for all sufficiently large $\alpha$.

Recalling that $\bar{P}(t)$ and $\bar{E}(\epsilon)$ commute, it follows from (3.7) that $v(t, \lambda)=\lambda^{\prime} \bar{E}^{1 / 2}(\epsilon) \bar{P}(t) \bar{E}^{1 / 2}(\epsilon) \lambda=\left\{\bar{E}^{1 / 2}(\epsilon) \lambda\right\}^{\prime} \bar{P}(t)\left\{\bar{E}^{1 / 2}(\epsilon) \lambda\right\}$. Eq. (2.8) of hypothesis (H3) now implies that

$$
c_{1}\left|\bar{E}^{1 / 2}(\epsilon) \lambda\right|^{2} \leq v(t, \lambda) \leq c_{2}\left|\bar{E}^{1 / 2}(\epsilon) \lambda\right|^{2}
$$

Schwarz's inequality implies that $\left|\bar{E}^{1 / 2}(\epsilon) \lambda\right| \leq\left|\bar{E}^{1 / 2}(\epsilon)\right| \lambda \mid$ and that

$$
|\lambda|=\left|\bar{E}^{-1 / 2}(\epsilon) \bar{E}^{1 / 2}(\epsilon) \lambda\right| \leq\left|\bar{E}^{-1 / 2}(\epsilon)\right|\left|\bar{E}^{1 / 2}(\epsilon) \lambda\right| .
$$

Therefore

$$
\left|\bar{E}^{1 / 2}(\epsilon) \lambda\right| \geq \frac{|\lambda|}{\left|\widetilde{E}^{-1 / 2}(\epsilon)\right|}
$$

Hence $v(t, \lambda)$ satisfles

$$
\frac{c_{1}}{\left|\bar{E}^{-1 / 2}(\epsilon)\right|^{2}}|\lambda|^{2} \leq v(t, \lambda) \leq c_{2}\left|\bar{E}^{1 / 2}(\epsilon)\right|^{2}|\lambda| .
$$

A further inequality which will be employed below is

$$
\left|\bar{E}^{-1 / 2}(\epsilon)\right|^{2}\left|\bar{E}^{1 / 2}(\epsilon)\right|^{2} \leq n^{2} \bar{K} .
$$

where $\bar{K}$ is given by

$$
\bar{K}:=\sum_{i=1}^{M} m_{i}^{2}+\sum_{i<j} m_{i} m_{j}\left(K_{i j}+K_{j i}\right)
$$

and the $K_{1 j}$ have been defined in Eq. (2.11) of (H4). The inequality (3.15) may be easily obtained from (2.11) if one recalls that $\left|\bar{E}^{ \pm 1 / 2}\right|=n^{1 / 2}\left|E^{ \pm 1 / 2}\right|$ since the matrix norm is the Frobenius norm. 
One now applies Proposition 1. Define the set $M(\beta)$ by

$$
M(\beta):=\left\{\lambda \in R^{m n}:|\lambda| \leq\left[\beta+\max \left(\left|\bar{D}^{-1}(0)\right||\bar{C}(0)|, 2|\bar{P}||\bar{C}| / c_{3}\right)\right]=: \alpha_{1}(\beta)\right\}
$$

where $\beta>0$ is arbitrary. Let the set $M_{+}(\beta)$ be deflned as

$$
M_{r}(\beta):=\left\{\lambda \in R^{m n}:|\lambda| \leq n \bar{K}^{1 / 2}\left(\frac{c_{2}}{c_{1}}\right)^{1 / 2} \alpha_{1}(\beta)=: \alpha_{2}(\beta)\right\}
$$

Using inequalities (3.14), (3.15) above it is not difficult to show that $\lambda_{1} \in M(\beta)$ and $\lambda_{2} \in M_{r}^{c}(\beta)$ implies that $v\left(t_{1}, \lambda_{1}\right)<v\left(t_{2}, \lambda_{2}\right)$ for all $t_{2} \geq t_{1} \geq 0$, for any $\epsilon \in H$.

Next an upper bound $\mu_{1}(\beta)$ on $\mu$ will be obtained such that $p_{\mu}(\alpha)<0$ for all $\alpha \in\left[\alpha_{1}(\beta), \alpha_{2}(\beta)\right]$ whenever $0<\mu<\mu_{1}(\beta)$. The existence of such an upper bound, along with the preceding conclusions, implies that Proposition 1 applies so that any solution of Eq. (3.5) with initial condition in $M(\beta)$ will remain in $M_{r}(\beta)$ for all $t \geq 0$ if $|\bar{E}(\epsilon)|<\mu_{1}(\beta)$. This will in turn imply the existence of solutions $L(t, \epsilon)$ to Eq. $(3.3)$ bounded by $\alpha_{2}(\beta)$ (since $|L|=|\lambda|$ ).

Proceeding, it is easy to see that (3.10) implies that for any $\alpha>2|\bar{P}||\bar{C}| / c_{3}, p_{\mu}(\alpha)<0$ for all $\mu \in\left[0, \mu^{*}(\alpha)\right)$ where

$$
\mu^{*}(\alpha):=\frac{c_{3} \alpha-2|\bar{P} \| \bar{C}|}{2 n^{1 / 2}|\bar{B}||\bar{P}| \alpha^{2}+\left\{\left|\frac{d}{d t} \bar{P}\right|+2 n^{1 / 2}|\bar{A}||\bar{P}|\right\} \alpha}
$$

From (3.17) and (3.18) it is clear that for any $\beta>0$ and any $\alpha \in\left[\alpha_{1}(\beta), \alpha_{2}(\beta)\right]$, one has $\mu^{*}(\alpha)>0$. Define

$$
\mu_{1}(\beta):=\min _{\alpha_{1}(\beta) \leq \alpha \leq \alpha_{2}(\beta)} \mu^{*}(\alpha)
$$

which is clearly positive for any $\beta>0$.

An application of Proposition 1 and Remark 2 now implies that for any $\beta>0$, all solutions of Eq. (3.5) with initial condition in $M(\beta)$ will remain in $M_{r}(\beta)$ for all $t \geq 0$, if $|\bar{E}(\epsilon)|<\mu_{1}(\beta)$. Noting that $M_{r}(\beta)$ is bounded and contains (by construction) the point $\lambda=-\bar{D}^{-1}(0) \bar{C}(0)$ (corresponding in Eq. (3.3) to $L=-D^{-1}(0) C(0)$ ) completes the proof of Theorem 1.

Q.E.D.

The preceding proof is constructive in that it also provides an explicit upper bound on $|E(\epsilon)|$ (actually on the related quantity $|\bar{E}(\epsilon)|$ ) ensuring the existence of bounded solutions to (3.3). Even more, it provides a family of upper bounds, one for each $\beta>0$. The next corollary summarizes these observations to give an 'optimal' upper bound on $|E(\epsilon)|$.

Corollary 1. Let (H1)-(H4) above hold, and moreover, suppose that both $B(t)$ and $C(t)$ have nonzero norms, as functions on $[0, \infty)$. Define $\beta_{0}$ as the largest real value of $\beta$ for which

$$
\mu^{*}\left(\alpha_{1}\left(\beta_{0}\right)\right)=\mu^{*}\left(\alpha_{2}\left(\beta_{0}\right)\right)
$$

and define $\beta^{*}$ as

$$
\beta^{*}:=\max \left(\beta_{0}, 0\right)
$$

Define $\alpha^{*}$ as

$$
\alpha^{*}:=\alpha_{2}\left(\beta^{*}\right)
$$

Then the upper bound $E_{1}$ on $|E(\epsilon)|$ in Theorem 1 may be taken as

$$
\begin{aligned}
E_{1} & :=n^{-1 / 2} \sup _{\beta>0}\left\{\mu_{1}(\beta)\right\} \\
& =n^{-1 / 2} \mu^{*}\left(\alpha^{*}\right) .
\end{aligned}
$$

Before proving this coroltary, it seems appropriate to make some remarks. 
Remark 3. In case $\beta^{*}=\beta_{0}$, one can clearly choose $\alpha^{*}=\alpha_{1}\left(\beta^{*}\right)$ rather than $\alpha_{2}\left(\beta^{*}\right)$ in Eq. (3.21c), since by (3.21a) $\mu^{*}$ will be independent of the choice.

Remark 4. It is easily verified that solving Eq. (3.21a) amounts to solving a cubic equation in the unknown $\beta_{0}$.

Remark 5. The cases $|B|=0$ and $|C|=0$ can be handled separately. If $|C|=0$, Eq. (3.3) clearly has the solution $L=0$ for any $\epsilon$, so that the upper bound $E_{1}$ may be set to $\infty$. If $|B|=0$ but $|C| \neq 0$, then Eq. (3.21a) does not have a solution. Instead, using Eq. (3.19) in Eq. (3.22a), one finds $E_{1}=n^{-1 / 2} c_{3}\left\{\left|\frac{d}{d t} \bar{P}\right|+2 n^{1 / 2}|\bar{A}||\bar{P}|\right\}^{-1}$ for this case.

The expressions (3.21b), (3.21c) above needed to compute $E_{1}$ can be made simpler if one is satisfied with finding an $E_{1}>0$ such that $|E(\epsilon)|<E_{1}$ implies the existence of some family of uniformly bounded solutions of the Riccati differential equation (3.3). The expressions above imply more: namely, the solution of (3.3) with initial condition $L(0, \epsilon)=-D^{-1}(0) C(0)$ is uniformly bounded for $|E(\epsilon)|<E_{1}$, as required in Theorem 1 . Since this requirement is unnecessary for many questions of decomposition and stability of (1.1), and indeed can lead to needlessly conservative bounds, the simpliffed results corresponding to Eqs. (3.21), (3.22) will now also be given.

Corollary 2. Let (H1)-(H4) above hold, and moreover, suppose that both $B(t)$ and $C(t)$ have nonzero norms, as functions on $[0, \infty)$. Define $\beta_{0}^{*}$ as the positive solution of

$$
\mu^{*}\left(\beta_{0}^{*}\right)=\mu^{*}\left(\alpha_{\ell}^{*}\left(\beta_{0}^{*}\right)\right)
$$

where

$$
\alpha_{Z}^{*}(\beta):=n \bar{K}^{1 / 2}\left(\frac{c_{2}}{c_{1}}\right)^{1 / 2} \beta
$$

Let $\alpha^{* *}$ denote the quantity

$$
\alpha^{* *}:=\text { either } \beta_{0}^{*} \text { or } \alpha_{2}^{*}\left(\beta_{0}^{*}\right)
$$

Let $E_{1}^{*}$ denote the quantity

$$
\begin{aligned}
E_{i}^{*} & :=n^{-1 / 2} \sup _{\beta>0}\left\{\mu_{1}(\beta)\right\} \\
& =n^{-1 / 2} \mu^{*}\left(\alpha^{* *}\right) .
\end{aligned}
$$

Then Eq. (3.3) has a solution $L(t, \epsilon)$ which is uniformly bounded for $|E(\epsilon)|<E_{t}^{*}, \epsilon \in H, t \geq 0$.

Proof of Corollary 1: Under the stated assumptions, a quick sketch of the graph of $\mu^{*}$ and some reflection shows that Eq. (3.21a) has a unique positive solution $\beta_{0}$ and that the corresponding value of $\alpha^{*}$ in Eq. (3.21c) achieves the equality of the right sides of (3.22a) and (3.22b). The conclusion now follows from the proof of Theorem 1 .

Proof of Corollary 2: The proof of Corollary 1 applies with only minor changes.

Next a proof will be given for Theorem 2. This proof relies on first ensuring the uniform asymptotic stability of the fast subsystem (3.2b) and then using an explicit representation for $M$ to prove uniform boundedness. Rather than assuming $|E(\epsilon)|<E_{1}$, the proof begins by assuming $|E(\epsilon)|<E_{i}^{*}$. This is sufficient, by Corollary 2, for the existence of uniformly bounded solutions $L(t, \epsilon)$ of the Riccati differential equation (3.3).

Proof of Theorem 2: Let $Y(t, s, \epsilon)$, respectively $Z(t, s, \epsilon)$, denote the state transition matrices of systems (3.2a) and (3.2b). Consider the variation of the Liapunov function candidate $w(t, \xi)$ defined by

$$
w(t, \xi):==\xi^{\prime} P(t) E(\epsilon) \xi
$$

along trajectories of Eq. (3.2b), the fast subsystem. Let $|E(\epsilon)|<E_{1}^{*}$, so that the foregoing proof 
of Theorem 1 and Corollary 2 imply $|L|<\alpha_{2}^{*}\left(\beta_{0}^{*}\right)$, where $\beta_{0}^{*}$ is the solution of Eq. (3.23a).

One has

$$
\begin{aligned}
\dot{w}(t, \xi) & =\xi^{\prime}\left\{D^{\prime}(t) P(t)+P(t) D(t)\right\} \xi \\
& +\xi^{\prime}\left\{B^{\prime}(t) L^{\prime}(t, \epsilon) P(t) E(\epsilon)+P(t) E(\epsilon) L(t, \epsilon) B(t)+\dot{P}(t) E(\epsilon)\right\} \xi \\
\leq & \left\{-c_{3}+|E(\epsilon)|\left(2|B||L||P|+\left|P^{\circ}\right|\right)\right\}|\xi|^{2} \\
\leq & \left\{-c_{3}+\nu\left(2 \alpha_{2}^{*}\left(\beta_{0}^{*}\right)|B||P|+|\dot{P}|\right)\right\}|\xi|^{2} \\
& =:-\bar{c}_{3}(\nu)|\xi|^{2}
\end{aligned}
$$

where the additional constraint

$$
|E(\epsilon)|<\nu \leq \frac{c_{3}}{2 \alpha_{2}^{*}\left(\beta_{0}^{*}\right)|B||P|+\left|P^{\prime}\right|}=: \nu^{*}
$$

has been imposed on $|E(\epsilon)|$, and $\nu$ is an auxiliary parameter. Eq. (3.27) ensures that $\bar{c}_{3}(\nu)$ of (3.26) above will be positive implying the null solution of Eq. (3.2b) is uniformly asymptotically stable.

Note that, by Eq. (2.8),

$$
\begin{aligned}
w(t, \xi) & =\left(E^{1 / 2}(\epsilon) \xi\right)^{\prime} P(t)\left(E^{1 / 2}(\epsilon) \xi\right) \\
& \leq c_{2}\left|E^{1 / 2}(\epsilon) \xi\right|^{2} \\
& \leq c_{2}\left|E^{1 / 2}(\epsilon)\right|^{2}|\xi|^{2} .
\end{aligned}
$$

Therefore, along trajectories of $(3.2 \mathrm{~b})$,

$$
|\xi|^{2} \geq \frac{w(t, \xi)}{c_{2}\left|E^{1 / 2}(\epsilon)\right|^{2}}
$$

Eq. (3.26) now implies the differential inequality

$$
\dot{w}(t, \xi) \leq-\frac{\bar{c}_{3}(\nu) w(t, \xi)}{c_{2}\left|E^{1 / 2}(\epsilon)\right|^{2}},
$$

so that $w(t, \xi)$ satisfies

$$
w(t, \xi(t)) \leq e^{-\frac{\bar{c}_{3}(\nu)}{\left.c_{2} E^{1 / 2}(t)\right|^{2}}(t-8)} w(s, \xi(s))
$$

for any $t \geq s \geq 0$. Recalling the definition (3.25) of $w$, this implies (by the Schwarz inequality)

$$
w(t, \xi) \leq|E(\epsilon)||P(s)||\xi(s)|^{2} e^{-\frac{\bar{c}_{3}(\nu)}{c_{2}\left|E^{1 / 2}(\epsilon)\right|^{2}}(t-8)}
$$

for any $t \geq s \geq 0$.

Eq. (2.8) of (H3) is now applied once more (the dependence of $E$ on $\epsilon$ is now suppressed):

$w(t, \xi)=\left(E^{1 / 2} \xi\right)^{\prime} P(t)\left(E^{1 / 2} \xi\right)$

$$
\begin{aligned}
& \geq c_{1}\left|E^{1 / 2} \xi\right|^{2} \\
& \geq \frac{c_{1}}{\left|E^{-1 / 2}\right|^{2}}|\xi|^{2} .
\end{aligned}
$$

Inequilities (3.32) and (3.33) together imply 


$$
|\xi(t)|^{2} \leq c_{1}^{-1}|P(s)||\xi(s)|^{2}|E| \mid E^{-1 / 2} P^{2} e^{-\frac{\bar{c}_{3}(\nu)}{\left.c_{2} E^{1 / 2}\right|^{2}}(t-s)}
$$

for all $t \geq s \geq 0$.

Next consider the implication of (3.34) for the state transition matrix $Z(t, s, \epsilon)$ of Eq. (3.2b). Since (3.34) is satisfied for each of the columns $Z^{i}$ of $Z$ and since $|Z|^{2}=\sum\left|Z^{i}\right|^{2}$ (Frobenius norm), and noting that $Z(s, s, \epsilon)=I$, one obtains the inequality

$$
|Z(t, s, \epsilon)|^{2} \leq c_{1}^{-1} \mid P(s)\|E\| E^{-1 / 2} p e^{-\frac{\bar{c}_{3}(\nu)}{c_{2} \mid E^{1 / 2}{ }^{1 / 2}}(t)}
$$

However, note that by the Schwarz inequality and (3.15)

$$
\begin{aligned}
|E|\left|E^{-1 / 2}\right| & =\left|E^{1 / 2} E^{1 / 2}\right|\left|E^{-1 / 2}\right|^{2} \\
& \leq\left|E^{1 / 2}\right|^{2}\left|E^{-1 / 2}\right|^{2} \\
& \leq \bar{K}^{2}
\end{aligned}
$$

where $\bar{K}$ was defined in (3.16). Eqs. (3.35) and (3.36) now imply

$$
|Z(t, s, \epsilon)| \leq \bar{K} c_{1}^{-1 / 2}|P(s)|^{1 / 2} e^{-\left.\frac{\bar{c}_{3}(\nu)}{2 c} e_{2} E^{1 / 2}\right|^{2}(t-8)}
$$

for all $t \geq s \geq 0$.

Note that the state transition matrix $Y(t, s, \epsilon)$ of Eq. (3.2a) satisfies

$$
|Y(t, s, \epsilon)| \leq \bar{Y}(\epsilon) e^{\sigma_{0}|t-s|}
$$

for some $\bar{Y}, \sigma_{0}>0$, since the coefficient matrix is bounded. Indeed, a specific $\sigma_{0}$ is given as

$$
\sigma_{0}:=|A|+|B| \alpha_{2}^{*}\left(\beta_{0}^{*}\right)
$$

where the function $\alpha_{g}^{*}(\beta)$ is defined in Eq. (3.23b) and $\beta_{0}^{*}$ has been defined above as the solution to Eq. (3.23a).

It can be verified by differentiation that

$$
M(t, \epsilon)=-\int_{t}^{\infty} Y(t, s, \epsilon) B(s) Z(s, t, \epsilon) d s \quad E^{-1}(\epsilon)
$$

is a solution of Eq. (3.4) (cf. Chang [35]). Using Eqs. (3.37) and (3.38), one now has

$$
\begin{aligned}
|M(t, \epsilon)| & \leq c_{1}^{-1 / 2} \bar{K} \bar{Y}(\epsilon)|B||P|^{1 / 2}\left|E^{-1}(\epsilon)\right| \int_{t}^{\infty} e^{\sigma_{0}(\theta-t)} e^{-\frac{\bar{c}_{3}(\nu)}{\left.\left.2 c_{2}\right|^{1 / 2}\right|^{2}(\theta-t)}} d s \\
& \leq c_{1}^{-1 / 2} \bar{K} \bar{Y}(\epsilon)|B||P|^{1 / 2}\left|E^{-1}(\epsilon)\right| \frac{e^{-\sigma(\nu, \epsilon) t}}{\sigma(\nu, \epsilon)} \lim _{T \rightarrow \infty}\left\{e^{\sigma(\nu, \epsilon) T}-e^{\sigma(\nu, \epsilon) t}\right\}
\end{aligned}
$$

where $\sigma(\nu, \epsilon)$ is defined as

$$
\sigma(\nu, \epsilon):=\sigma_{0}-\frac{\bar{c}_{3}(\nu)}{2 c_{2}\left|E^{1 / 2}(\epsilon)\right|^{2}} .
$$

Therefore $M(t, \epsilon)$ is bounded for all $\epsilon$ for which $\sigma(\nu, \epsilon)<0$, which is equivalent to the inequality

$$
\left|E^{1 / 2}(\epsilon)\right|^{p}<\frac{\bar{c}_{3}(\nu)}{2 \sigma_{0} c_{2}} .
$$


Eq. (3.43) can be used to yield an upper bound on $|E(\epsilon)|$ as follows. Suppose $\epsilon$ were constrained so that $|E(\epsilon)|^{2}=m_{1} \epsilon_{1}{ }^{2}+\ldots+m_{M} \epsilon_{M}{ }^{2}<k$ where $k_{M}>0$ is arbitrary. This would clearly imply $\epsilon_{i}<\left(k / m_{i}\right)^{1 / 2}, i=1, \ldots, M$, so that $\mid E^{1 / 2}(\epsilon)^{2}=\left(\sum_{i=1}^{M} m_{i} \epsilon_{i}\right)^{2}<k\left(\sum_{i=1}^{M} m_{i}{ }^{1 / 2}\right)^{2}$. Equating the right side of this last inequality with the right side of (3.43) and solving for $k$ shows that (3.43) is implied by the following upper bound on $|E(\epsilon)|^{2}$ :

$$
|E(\epsilon)|^{2}<\frac{\bar{c}_{3}(\nu)}{2 \sigma_{0} c_{2}}\left(\sum_{i=1}^{M} m_{i}{ }^{1 / 2}\right)^{-2}
$$

Note that the auxiliary parameter $\nu$ is still arbitrary subject to $0<\nu<\nu^{*}$ (Eq. (3.27)). To optimize the upper bound, one maximizes the lesser of the two upper bounds imposed by Eqs. (3.27) and (3.44). Thus it is required that

$$
|E(\epsilon)|<E_{2}
$$

where the upper bound $E_{2}$ is given by

$$
E_{2}:=\max _{0 \leq \nu \leq \nu^{*}} \min \left\{\nu,\left(\frac{\bar{c}_{3}(\nu)}{2 \sigma_{0} c_{2}}\right)^{1 / 2}\left(\sum_{i=1}^{M} m_{i}{ }^{1 / 2}\right)^{-1}\right\}
$$

Finally, the uniform boundedness of $M(t, \epsilon)$ can be shown by applying the inequality (3.15) to obtain an upper bound on (3.41). Such an upper bound will not be derived here. This is because the uniform boundedness of $M(t, \epsilon)$ will not be needed for the stability considerations of this paper, and since ensuring uniform boundedness of $M(t, \epsilon)$ would require a further constraint on $|E(\epsilon)|$.

Q.E.D.

\section{III.2. Uniform Asymptotic Stability}

The decoupling transformation (3.1) is invertible for any $\epsilon$. Indeed, it is easy to check that the inverse transformation is given by

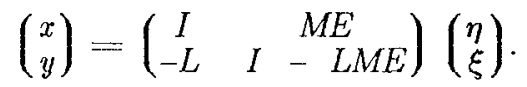

From (3.1) and (3.47) it is clear that for bounded $L(t, \epsilon), M(t, \epsilon)$, the uniform asymptotic stability of (1.1) is equivalent to the uniform asymptotic stability of (3.2a) and (3.2b).

An upper bound on $|\epsilon|$ ensuring uniform asymptotic stability of the fast subsystem (3.2b) has been derived in the foregoing analysis. It remains to find an upper bound ensuring the uniform asymptotic stability of the slow subsystem (3.2a).

It is useful to state the following basic proposition, whose proof (essentially) may be found in Brockett [39, p. 205].

Proposition 2. Let the null solution of $\dot{x}(t)=A(t) x(t)$ be uniformly asymptotically stable, and let $v(x):=x^{\prime} R(t) x \quad$ be a Liapunov function with $S(t):=$ $-\left(\dot{R}(t)+A^{\prime}(t) R(t)+R(t) A(t)\right)$ positive definite. Then the null solution of the equation $\dot{x}=[A(t)+B(t)] x$ is also uniformly asymptotically stable for any $B(t)$ with $|B|<\delta$ where $\delta$ is given $b y$

$$
\delta:=\frac{|S|}{2|R|}
$$

a necessarily positive quantity.

To apply this proposition to Eq. (3.2a), it is necessary to find an estimate for the difference between the coefficient matrix in (3.2a) and the reduced system matrix $A_{0}(t)$ of Eq. (2.3). This estimate should depend on $\epsilon$, and moreover vanish in the limit $\epsilon \rightarrow 0, \epsilon \in H$. The following mild nypothesis will be used in the derivation. 
(H5) The matrix $D^{-1}(t) C(t)$ is continuously differentiable on $[0, \infty)$.

Defining the vector $l \in R^{m n}$ as

$$
l:=\lambda-\bar{D}^{-1}(t) \bar{C}(t)
$$

Eq. (3.5) can be rewritten as

$$
\begin{aligned}
\bar{E}(\epsilon) l(t)=-\bar{E}(\epsilon) \frac{d}{d t}\left\{\bar{D}^{-1}(t) \bar{C}(t)\right\}+\bar{D}(t) l(t) & -\bar{E}(\epsilon) \bar{L}(t) \bar{A}(t) \\
& +\bar{E}(\epsilon) \bar{L}(t) \bar{B}(t) \lambda(t) .
\end{aligned}
$$

As in the proof of Theorem 1, the Liapunov function candidate

$$
v(t, l)=l^{\prime} \bar{P}(t) \bar{E}(\epsilon) l
$$

is introduced. Evaluating $\dot{v}(t, l)$ along trajectories of (3.50) and proceeding as in the proof of Theorem 1, one obtains

$$
\begin{aligned}
\dot{v}(t, l) \leq & -c_{3}|l|^{2}+\left|\frac{d}{d t} \bar{P}\right||\bar{E}||l|^{2}+2|\bar{A}||\bar{P}||\bar{L}||\bar{E}||l| \\
& +2|\bar{P}|\left|\frac{d}{d t}\left(\bar{D}^{-1} \bar{C}\right)\right||\bar{E}||l|+2|\bar{P}||\bar{B}||\bar{L}||\lambda||\bar{E}||l|
\end{aligned}
$$

From (3.49) it follows that $|\lambda| \leq|l|+\left|\bar{D}^{-1} \bar{C}\right|$, so that $|\bar{L}| \leq n^{1 / 2}\left(|l|+\left|\bar{D}^{-1} \bar{C}\right|\right)$. Using these facts, inequality (3.52) may be strengthened to

$$
\dot{v}(t, l) \leq-\gamma(\kappa)|l|^{2}+\rho_{0} \kappa|l|+\rho_{2} \kappa||^{3}
$$

for $|E(\epsilon)|<\kappa$, where

$$
\gamma(\kappa):=c_{3}-\rho_{1} \kappa
$$

where

$$
\begin{aligned}
& \rho_{0}:=2 n^{1 / 2}|\bar{P}|\left|\frac{d}{d t}\left(\bar{D}^{-1} \bar{C}\right)\right|+2 n|\bar{P}||\bar{B}|\left|\bar{D}^{-1} \bar{C}^{2}+2 n\right| \bar{A}|| \bar{P}|| \bar{D}^{-1} \bar{C} \mid, \\
& \rho_{1}:=n^{1 / 2}\left|\frac{d}{d t} \bar{P}\right|+2 n|\bar{A}||\bar{P}|+4 n|\bar{B}||\bar{P}|\left|\bar{D}^{-1} \bar{C}\right|, \\
& \rho_{2}:=2 n|\bar{P}||\bar{B}|
\end{aligned}
$$

and $\kappa$ is an auxiliary parameter.

Application of Proposition 1 in a fashion similar to that in the proof of Theorem 1 now yields the following result, which is stated for Eq. (3.3) for convenience.

Theorem 3. Let (H1)-(H5) above hold. Then for any $\delta>0$ there exists a bounded solution $L(t, \epsilon)$ to $E q$. (3.3) with $\left|L(t, \epsilon)-D^{-1}(t) C^{\prime}(t)\right|<\delta, t \geq 0$ whenever $|E(\epsilon)|<\kappa^{*}(\delta)$ where $\kappa^{*}(\delta)$ is given by

$$
\kappa^{*}(\delta):=\min _{\alpha_{0} \delta \leq \alpha \leq \delta} \frac{n^{-1 / 2} c_{3} \alpha}{\rho_{0}+\rho_{1} \alpha+\rho_{2} \alpha^{2}}
$$

in which

$$
\alpha_{0}:=n^{-1} \bar{K}^{-1 / 2}\left(\frac{c_{1}}{c_{2}}\right)^{1 / 2} .
$$

Proof: The theorem follows by applying Proposition 1 to Eq. (3.50) using the Liapunov thitiuniti 
$v$ of (3.51) and the estimate (3.53) on $\dot{v}$. Define $M_{r}$ of Proposition 1 as $\left\{l \in R^{m n}:|l|<\delta\right\}$. Inequalities (3.14), (3.15) imply that the set $M$ defined by $M:=\left\{l \in R^{m n}\right.$ : $\left.|l|<n^{-1} \bar{K}^{-1 / 2}\left(c_{1} / c_{2}\right)^{1 / 2} \delta\right\}$ and $M_{r}$ flt the mold of Proposition 1. Eq. (3.53) is now used to show that if $|E(\epsilon)|\left(=n^{-1 / 2}|E(\epsilon)|\right)<\kappa^{*}(\delta)$, then $\dot{v}(t, l)<0$ on $M-M_{r}$. Proposition 1 and Remark 2 now assert the existence of a solution $l(t, t)$ of $(3.50)$ with $|l|<\delta$. Recalling the oneto-one correspondence of solutions $l$ of (3.50) and solutions $L$ of (3.3) (see Eq. (3.49) and the definition of $\lambda$ following (3.5)) completes the proof.

The reduced system (2.3) is now assumed uniformly asymptotically stable.

(H6) The null solution of the reduced system (2.3) is uniformly asymptotically stable.

To apply Proposition 2, note that (H4) implies the reduced system (2.3) has a quadratic Liapunov function $v(x)=x^{\prime} R_{0}(t) x$ with $\dot{v}(x)=-x^{\prime} S_{0}(t) x<0$ along trajectories of $\dot{x}=A_{\mathrm{o}}(t)$ (Eq. (2.3)). For a proof of this standard result and an explicit formula for $R_{\mathrm{o}}(t)$ given any positive definite $S_{0}(t)$, see for instance Brockett [39, Theorem 6, p. 203]. Choosing a bounded $S_{0}(t)$, Proposition 2 and Theorem 3 now imply that the slow subsystem (3.2a) will be uniformly asy mptotically stable if

$$
|E(\epsilon)|<\kappa^{*}\left(\delta_{0}\right)
$$

where

$$
\delta_{\mathrm{o}}:=\frac{\left|S_{\mathrm{o}}\right|}{2\left|R_{\mathrm{o}}\right|}
$$

Define $E_{3}$ as

$$
E_{3}:=\kappa^{*}\left(\delta_{0}\right)
$$

A parameter bound for the stability of time-varying multiparameter singular perturbation problems may now be stated.

Theorem 4: Let hypotheses (H1)-(H6) hold. Then the null solution of the multiparameter singularly perturbed system (1.1) is uniformly asymptotically stable for all $\epsilon \in H$ with $\left|E_{(}(\epsilon)\right|<E_{0}$ where $E_{0}$ is the positive scalar given by

$$
E_{0}:=\min \left(E_{1}^{*}, E_{2}, E_{3}\right)
$$

and $E_{1}^{*}, E_{2}, E_{3}$ are given in Eqs. (3.24b), (3.46) and (3.61), respectively.

\section{III.4. An Example}

It is instructive to consider an example illustrating the various computations involved in arriving at the bounds $E_{1}^{*}, E_{2}, E_{3}$ and the stability bound $E_{0}$. It is also of interest to compare the bound for stability $E_{0}$ with previously obtained upper bounds for time-varying singularly perturbed systems. In his dissertation [13], Saberi has obtained an upper bound on the small parameter for stability of a time-varying singularly perturbed system. (The corresponding results for time-invariant singularly perturbed systems appeared in Saberi and Khalil [14]. ) Saberi [13] presents argumentation supporting the conclusion that his upper bound is usually less conservative than previously obtained estimates. For the example considered below, the parameter bound for stability $E_{0}$ of this paper will be compared with that implied by the results of Saberi. ${ }^{1}$ Since the derivation of Saberi's bound does not involve separation of time scales, one might suspect that the stability bound for time-varying systems derived here will often be more conservative than that of [13]. This happens to be the case for the particular example presented below.

\footnotetext{
'A revlewer suggested that such a comparison with Saberl's bound be Included. It should be noted, however, that the relative tightness ol any two bounds can not ve judged on the basis of an example.
} 
Consider a singular perturbation problem (1.1) with $n=1, M=1, m=2$, and with matrices $A, B, C, D$ given by

$$
\begin{aligned}
A(t) & =\left(0.4 e^{-t}\right), & B(t) & =\left(\begin{array}{ll}
0 & 1
\end{array}\right), \\
C(t) & =\left(\begin{array}{l}
1 \\
0
\end{array}\right), & D(t) & =\left(\begin{array}{cc}
0 & 5\left(1+2 e^{-t}\right)^{-1} \\
-1 & -\left(3+e^{-t}\right)\left(1+2 e^{-t}\right)^{-1}
\end{array}\right) .
\end{aligned}
$$

One computes

$$
\begin{aligned}
& D^{-1}(t)=0.2\left(\begin{array}{cc}
-3-e^{-t} & -5 \\
1+2 e^{-t} & 0
\end{array}\right), D^{-1}(t) C(t)=0.2\left(\begin{array}{c}
-3-e^{-t} \\
1+2 e^{-t}
\end{array}\right), \\
& \frac{d}{d t} D^{-1}(t) C(t)=\left(\begin{array}{c}
0.2 e^{-t} \\
-0.4 e^{-t}
\end{array}\right) .
\end{aligned}
$$

Hence the reduced system is

$$
\dot{x}=A_{0}(t) x=-0.2 x \text {. }
$$

Choosing $Q(t)=2 I$ in Eq. (2.9) of hypothesis (H3), one computes $P(t)$ as

$$
P(t)=\left(\begin{array}{cc}
1+e^{-t} & 1 \\
1 & 2
\end{array}\right)
$$

which is easily seen to be positive definite. Also,

$$
c_{3}=2
$$

since $y^{\prime} Q y=2|y|^{2}$, and one can easily check that

$$
c_{1}=\frac{3-5^{1 / 2}}{2}=0.3820, c_{2}=3
$$

by taking limits as $t \rightarrow \infty$ and 0 respectively in $y^{\prime} P(t) y$ and completing the squares. The following quantities are now readily computed.

$$
\begin{aligned}
& |A|=0.4,|B|=1,|C|=1,|D|=(35)^{1 / 2}=5.9161, \\
& |P|=10^{1 / 2}=3.1623, \bar{K}=4,\left|D^{-1}(0)\right|=2^{1 / 2}=1.4142, \\
& \left|D^{-1} C\right|=1,\left|\frac{d}{d t} D^{-1} C\right|=0.2(5)^{1 / 2}=0.4472,\left|P^{*}\right|=1 .
\end{aligned}
$$

Now $\mu^{*}(\alpha)$ is approximately

$$
\mu^{*}(\alpha)=\frac{2 \alpha-6.324}{6.324 \alpha^{2}+3.5298 \alpha}
$$

and $\alpha_{1}(\beta), \alpha_{2}(\beta)$ are

$$
\begin{gathered}
\alpha_{1}(\beta)=\beta+\max (1.4142,3.1623)=\beta+3.1623 \\
\alpha_{2}(\beta)=5.605 \alpha_{1}(\beta)=5.605 \beta+17.7248
\end{gathered}
$$

Equating $\mu^{*}\left(\alpha_{1}(\beta)\right)$ to $\mu^{*}\left(\alpha_{2}(\beta)\right)$ and solving for $\beta$ gives

$$
\beta^{*}=0.6467
$$

Hence

$$
\alpha^{*}=3.8090
$$

so that 


$$
E_{1}=\mu^{*}\left(\alpha^{*}\right)=0.0123
$$

It is easy to check that solving Eq. (3.23a) and proceeding as in Corollary 2 to evaluate $E_{1}^{*}$ produces the same upper bound for this phase, in this particular example. That is, $E_{1}^{*}=E_{1}$ for this example.

Now $\sigma_{0}$ of Eq. (3.39) is

$$
\sigma_{0}=21.7494
$$

Also, one has

$$
\bar{c}_{3}(\nu)=2-136.0257 \nu
$$

and

$$
\nu^{*}=0.0147
$$

Now $E_{2}$ is merely the positive value of $\nu$ for which the two quantities in braces in Eq. (3.46) are equal. (It is easily verifled that exactly one such solution always exists, and that it is guaranteed less than $\nu^{*}$.) Solving the associated quadratic equation gives

$$
E_{2}=0.0143 \text {. }
$$

In the computation of $E_{3}$ one finds that $\delta_{0}=0.2$ (since the reduced matrix $A_{0}$ is simply - o.2). Finally, $\kappa^{*}(\delta)$ is given by

$$
\kappa^{*}(\delta)=\min _{0.0357 \leq \alpha \leq 0.2} \frac{2 \alpha}{3.1623+16.0734 \alpha+6.3246 \alpha^{2}}
$$

Graphing the ratio in (3.79) shows that the minimum must occur either at the left or right endpoint. In this case it occurs at the left endpoint, $\alpha=0.0357$, with the value

$$
E_{3}=\kappa^{*}\left(\delta_{0}\right)=0.0191 \text {. }
$$

Since $E_{1}^{*}$ is smaller than both $E_{2}$ (Eq. (3.78)) and $E_{3}$ (Eq. (3.80)), the parameter bound for stability $E_{0}$ based on the foregoing results is $E_{i}^{*}=E_{1}$, i.e.

$$
E_{0}=0.0123 \text {. }
$$

Saberi [13] has obtained an upper bound on the small parameter $\epsilon$ of linear time-varying singularly perturbed systems using composite Liapunov functions. The specialization of that result to the time-invariant case has appeared in Saberi and Khalil [14]. Applying the upper bound computation of [13] to this example gives an upper bound of

$$
\epsilon_{\mathrm{o}}=0.1953
$$

which is less conservative than the bound $E_{0}$ (Eq. (3.81)) obtained by the methods of this paper. (Note: In arriving at the bound $\epsilon_{0}$ of $(3.82)$, the solution $P(t)$ of the Liapunov matrix equation (2.9) given in Eq. (3.66) was employed.)

\section{TIME-INVARLANT PROBLEMS}

\section{IV.1. Motivation and Background}

\section{A. Motivation}

The remainder of the paper will be concerned with obtaining specialized results for the time-invariant version of Eq. (2.1):

$$
\begin{aligned}
\dot{x} & =A x+B y \\
E(\epsilon) \dot{y} & =C x+D y .
\end{aligned}
$$

The notation here is the same as that used for the time-varying case, except that now $A, B, C, D$ are constant matrices. The main reason for considering this special case separately is to show 
that hypothesis (H4) can be lifted for time-invariant problems. Another fortunate outcome of this separate analysis is that hypothesis (H3) can be replaced by a more natural assumption, namely the 'strong block D-stability' of an associated boundary layer system, a concept introduced below. It is interesting to note that the assumption ( $\mathbf{H 4 )}$ of bounded mutual ratios for the small parameters was crucial in the derivation of the upper bounds in the time-varying case, as was hypothesis (H3) on the matrix $D(t)$. Indeed, it is easily verifled that the upper bound for stability of Eq. (1.1) obtained above vanishes in the limit that the constraint (2.11) on the mutual ratios disappears. This can be checked by taking the limit as $\bar{K} \rightarrow \infty$ in (3.18), (3.20) to get $E_{1}^{*} \rightarrow 0$, implying $E_{0} \rightarrow 0$ by Eq. (3.62). The upper bound for stability obtained by Khalil [11] in the (nonlinear) time-invariant case suffers from this same type of conservativeness.

Three problems are dealt with in the remainder of the paper. The first problem is that of finding sufficient conditions ensuring asymptotic stability of the null solution of (4.1) for all sufficiently small $|\epsilon|, \epsilon \in R_{+}^{M}$. (Recall that $R_{+}^{M}$ is the positive orthant of $R^{M}$.) The second concerns finding upper bounds on the small parameters ensuring the existence of a transformation separating fast and slow dynamics of (4.1). For brevity, such an upper bound will be derived only to ensure that the system equations can be made block upper triangular, this being all that is needed to ensure stability in the time-invariant case. Ensuring the existence of an actual decoupling transformation can be achieved using the same methods. The third problem is, given that a set of sufficient conditions for asymptotic stability are indeed satisfled, to exhibit a specific upper bound $E_{0}$ on $|E(\epsilon)|$ such that $|E(\epsilon)|<E_{0}, \epsilon \in R_{+}^{M}$ implies the asymptotic stability of (4.1). Note that the small parameters are not constrained to lie in a proper subset of $R_{+}^{M}$; all that is assumed is $\epsilon_{i}>0, i=1, \ldots, M$. Thus the results will apply uniformly as $\epsilon \rightarrow 0$ along any path in $R_{+}^{M}$.

\section{B. Asymptotic Fast-Slow Decomposition and Stability Analysis}

The next lemma was introduced in [27]. It gives an algebraic matrix Riccati equation whose solution is useful in exhibiting a transformation which decouples the fast and slow modes of (4.1).

Lemma 1 [27]. Suppose det $D \neq 0$ and denote $E=E(\epsilon)$. Then the Riccati equation

$$
D \Gamma+E\left(D^{-1} C-\Gamma\right) A_{0}-E \Gamma B \Gamma+E D^{-1} C B \Gamma=0
$$

for the $m \times n$ matrix $\Gamma$ has a locally unique solution $\Gamma(\epsilon)$ near $0 \in R^{m \times n}$ for $|\epsilon|$ sufficiently small.

The Riccati equation (4.2) is related to the steady-state version of the differential matrix Riccati equation (3.3) for $L$ used in the decoupling of time-varying systems. Here it is convenient to deal with $\Gamma:=D^{-1} C-L$ rather than $L$ as the unknown, as $\Gamma=O(|\epsilon|)$.

The eigenvalues of (4.1) are of course the eigenvalues of the Jacobian matrix

$$
J(\epsilon)=\left(\begin{array}{cc}
A & B \\
E^{-1}(\epsilon) C & E^{-1}(\epsilon) D
\end{array}\right)
$$

of (1.1).

The next theorem gives an exact expression for the eigenvalues of $J(\epsilon)$ in terms of the eigenvalues of matrices associated with appropriate fast and slow subsystems of (4.1). This theorem was derived in $[27]$ by using Lemma 1 above to exhibit a similarity transformation rendering $J(\epsilon)$ in block upper triangular form.

Theorem 5. Let det $D \neq 0$ and let $|\epsilon|$ be sufficiently small so that Lemma 1 applies. Then

$$
\sigma(J(\epsilon))=\sigma\left(A-B D^{-1} C+B \Gamma(\epsilon)\right) \cup \sigma\left(E^{-1}(\epsilon) D+D^{-1} C B-\Gamma(\epsilon) B\right)
$$

if $\epsilon_{i} \neq 0, i=1, \ldots, M$.

This result motivates the following definition of fast and slow subsystems associated with (4.1). 
Definition 3. If $D$ is nonsingular and a solution $\Gamma(\epsilon)$ of Eq. (4.2) exists, then the corresponding slow subsystem of (4.1) is

$$
\dot{x}=\left(A-B D^{-1} C+B \Gamma(\epsilon)\right) x,
$$

and the fast subsystem is

$$
\dot{y}=\left(E^{-1}(\epsilon) D+D^{-1} C B-\Gamma(\epsilon) B\right) y .
$$

Several results on the stability of Eq. (4.1) can be obtained from the foregoing discussion. The first follows from a time-varying version proved by Khalil and Kokotovic [16]. It applies under the bounded mutual ratios assumption, and can be proved readily using Theorem 5 [29].

Theorem 6. Let hypothesis (H4) apply, so that $\epsilon \in H$ and $H$ is a cone of the form (2.11). Then the null solution of (4.1) is asymptotically stable for all $\epsilon \in H$ with $|\epsilon|$ sufficiently small if: (i) the reduced system obtained by formally setting $\epsilon=0$ is asymptotically stable, i.e.

$$
\operatorname{Re} \sigma\left(A-B D^{-1} C\right)<\mathbf{0}
$$

and (ii)

$$
\operatorname{Re} \sigma\left(E^{-1}(\epsilon) D\right)<0
$$

for all $\epsilon \in H$.

Condition ( $i i$ ) of Theorem 6 holds if $D$ is block $D$-stable relative to the multi-index $\left(m_{1}, \ldots, m_{M}\right)$. Before stating the next theorem it is useful to introduce the following terminology $[29]$.

Definition 4. The matrix $F \in R^{m \times m}$ is strongly D-stable if there is a $\mu>0$ such that $F+G$ is D-stable for each $G \in R^{m \times m}$ with $|G|<\mu$.

Definition 4 is a special case of the following more general notion of 'strong block Dstability.' In [29] it has been shown that matrices satisfying hypothesis (H3) with constant positive definite matrices $P, Q$ are strongly block D-stable.

Definition 5. The matrix $F \in R^{m \times m}$ is strongly block $D$-stable (relative to the multi-index $\left.\bar{m}:=\left(m_{1}, \ldots, m_{M}\right)\right)$ if there is a $\mu>0$ such that $F+G$ is block D-stable (relative to $\bar{m}$ ) for each $G \in R^{m \times m}$ with $|G|<\mu$.

The next theorem states that if the reduced system is stable and if $D$ is strongly block Dstable, stability of the multiparameter singular perturbation problem (4.1) is guaranteed for all sufficiently small $|\epsilon|, \epsilon \in R_{+}^{M}$. Note the removal of the constraint $(2.11)$ on the relative magnitudes of the singular perturbation parameters which was needed in Theorem 6 . Thus, assuming strong block D-stability, a significant generalization is realized. Both the two time-scale setting and the multiple time-scale case are treated in the same framework. Strong block D-stability is also very natural in that it forms part of a necessary and sufficient condition for the robust stability of Eq. (4.1) $\{30\}$.

Theorem 7. Suppose that all eigenvalues of $A_{0}=A-B D^{-1} C$ have strictly negative real parts, and let $D$ be strongly block $D$-stable, relative to the multi-index $\left(m_{1}, \ldots, m_{M}\right)$. Then there is a $\mu>0$ such that the null solution of system (4.1) is asymptotically stable for all $\epsilon=\left(\epsilon_{1}, \ldots, \epsilon_{M}\right)$ with $|\epsilon|<\mu$ and $\epsilon_{i}>0, i=1, \ldots, M$.

Proof: The slow subsystem (4.5) will clearly be asymptotically stable for sufficiently small $|\epsilon|$. Also, since $D$ is strongly block $D$-stable, the matrix

$$
D+E(\epsilon) D^{-1} C B-E(\epsilon) \Gamma(\epsilon) B
$$

will also be $D$-stable for sufficiently small $|\epsilon|$. Premultiplying by $E^{-1}(\epsilon)$, a block diagonal matrix of the appropriate structure, necessarily yields a stable matrix. This stable matrix is precisely the Jacobian of the right side of the fast subsystem equation (4.6). proof.

Theorem 7 is a special case of the following result, whose proof is analogous to the preceding 
Theorem 8. Suppose $A_{0}$ is a stable matrix, and let the set $H \subset R_{+}^{M}$ be such that

$$
\operatorname{Re} \sigma\left(E^{-1}(\epsilon) D\right)<0
$$

for all $\epsilon \in H$. Moreover, assume that (4.10) also holds if $D$ is replaced by any sufficiently small perturbation of $D$, for all $\epsilon \in H$. Then the null solution of (4.1) is asymptotically stable for all $\epsilon \in H$ with $|k|$ sufficiently small.

\section{Strategy}

The strategy which will be followed in the remainder of the paper may now be briefly summarized. The derivation consists of two main steps. First, the Brouwer fixed point theorem is used to obtain an initial upper bound $E_{1}$ on $|E(\epsilon)|$ which ensures the existence of a solution to the algebraic matrix Riccati equation (4.2). This initial upper bound is parametrized by the magnitude of a norm constraint imposed on a solution to the Riccati equation. Satisfaction of this upper bound then ensures that the fast and slow subsystems introduced above are meaningful, and moreover that asymptotic stability of (4.1) is equivalent to that of the fast and slow subsystems. Next, hypothesis (H3) is invoked. This facilitates application of Liapunov's direct method to obtain a bound on the norm of the solution to the Riccati equation which ensures asymptotic stability of the fast and slow subsystems. An explicit upper bound on the weighted norm $|E(\epsilon)|$ of the vector of singular perturbation parameters $\epsilon$ readily follows. Note that a similar approach was used by this author in [7] to obtain a parameter bound ensuring the stability of timeinvariant singularly perturbed systems containing a single parameter.

\section{IV.2. Fixed-Point Analysis of the Riccati Equation}

Rewrite Eq. (4.2) in the fixed point form

$$
F_{\epsilon}(\Gamma)=\Gamma
$$

where the parametrized mapping $F_{\epsilon}: R^{m \times n} \rightarrow R^{m \times n}$ is defined for any $\epsilon \in R^{M}$ by

$$
F_{\epsilon}(\Gamma):=D^{-1} E(\epsilon)\left\{\left(\Gamma-D^{-1} C\right) A_{0}+\Gamma B \Gamma-D^{-1} C B \Gamma\right\} .
$$

A parametrized upper bound $E_{1}(\alpha)$ on $|E(\epsilon)|$ will now be derived such that for any $\alpha>0$ and if $|E(\epsilon)|<E_{1}(\alpha),|\Gamma| \leq \alpha$ implies $\left|F_{\epsilon}(\Gamma)\right| \leq \alpha$. Proceeding, let $\alpha>0$ be given and let $\Gamma \in R^{m \times n}$ satisfy $|\Gamma| \leq \alpha$. From (4.12) one has

$$
\begin{aligned}
\left|F_{\epsilon}(\Gamma)\right| & \leq|E(\epsilon)|\left|D^{-1}\right|\left\{\left(|\Gamma|+\left|D^{-1} C\right|\right)\left|A_{0}\right|+|B||\Gamma|^{2}+\left|D^{-1} C B\right| \Gamma \mid\right\} \\
& \leq|E(\epsilon)|\left|D^{-1}\right|\left\{\left(\alpha+\left|D^{-1} C\right|\right)\left|A_{0}\right|+\alpha^{2}|B|+\alpha\left|D^{-1} C B\right|\right\} .
\end{aligned}
$$

From (4.13) follows immediately that for $\left|F_{\epsilon}(\Gamma)\right| \leq \alpha$ to hold it suffices that $|E(\epsilon)| \leq E_{1}(\alpha)$ where $E_{1}(\alpha)$ is given by

$$
E_{1}(\alpha):=\frac{\alpha}{\left|D^{-1}\right|\left\{\left|D^{-1} C\right|\left|A_{0}\right|+\alpha\left(\left|D^{-1} C B\right|+\left|A_{0}\right|\right)+\alpha^{2}|B|\right\}} .
$$

Thus for any $\alpha>0, F_{\epsilon}$ is a continuous map of the closed ball $B_{\alpha}:=\left\{\Gamma \in R^{m \times n}:|\Gamma| \leq \alpha\right\}$ into itself whenever $0 \leq \epsilon \leq E_{1}(\alpha)$. Note that $B_{\alpha}$ is homeomorphic to the closed unit ball in $R^{m n}$. The Brouwer fixed point theorem $[40$, p. 54], [41, p. 10] may now be invoked to establish the existence of a solution $\Gamma(\epsilon)$ to (4.11) in $B_{\alpha}$ for any $\epsilon$ with $|E(\epsilon)|<E_{1}(\alpha)$.

Theorem 9. For any $\alpha>0$ and for all $\epsilon \in R^{M}$ with $|E(\epsilon)| \leq E_{1}(\alpha)$, the Riccati equation (4.2) has at least one solution $\Gamma(\epsilon)$ with $|\Gamma(\epsilon)| \leq \alpha$.

Remark 6. Note that for any system (1.1) with $B \neq 0$ (the generic and nontrivial case), the upper bound $E_{1}(\alpha)$ approaches 0 as $\alpha \rightarrow \infty$. It is clear, however, that a solution of (1.2) in the ball $B_{\alpha}$ for a given $\alpha=\alpha_{0}$ will also belong to any ball $B_{\alpha}$ with $\alpha>\alpha_{0}$. Therefore the upper bound of Theorem 2 can be made less conservative by using instead of $E_{1}(\alpha)$ the revised estimate 


$$
E_{1}^{*}(\alpha):=\max _{0 \leq \beta \leq \alpha} E_{1}(\beta)
$$

Remark 7. It is natural to attempt to factorize the quadratic appearing in the denominator of the expression (4.14) for $E_{1}(\alpha)$. In this regard, note that replacing $\left|D^{-1} C B\right|$ in (4.14) by $\left|D^{-1} C\right||B|$ results in the new (in general more conservative) upper bound $E_{1}^{* *}$ given by

$$
E_{i}^{* *}(\alpha):=\frac{\alpha}{\left|D^{-1}\right|\left(\left|D^{-1} C\right|+\alpha\right)\left(\left|A_{\mathrm{o}}\right|+\alpha|B|\right)} .
$$

Remark 8. Note that in the derivation of Theorem 9 only existence of a solution $\Gamma$ to (4.2) needed to be ensured. A further upper bound on $|E(\epsilon)|$ to ensure uniqueness could easily be derived by a contraction mapping argument as in [5, pp. 16-18]. However, this could only result in a more conservative final estimate and would thus be counterproductive.

\section{IV.3. An Upper Bound for Stability}

\section{A. A Robustness of Stability Estimate}

In the stability analysis of the slow subsystem (4.5) the specialization of Proposition 2 to the time-invariant case will prove useful. It is restated below in a form suitable for time-invariant systems.

Proposition 2(Time-Invariant Case). Let $A$ be a stable matrix. Assume given a positive definite matrix $T$ and the (unique) positive definite solution $R$ of the Liapunov matrix equation $A^{\prime} R+R A=-T$. Then $A+B$ will be a stable matrix for any $B$ with $|B|<\delta$, where $\delta$ is given by

$$
\delta:=\frac{|T|}{2|R|}
$$

It is worth noting that the matrix $R$ has the explicit representation

$$
R=\int_{0}^{\infty} e^{A^{\prime} t} T e^{A t} d t
$$

even though this in general does not indicate an efficient means of computing $R$ (cf. Laub [42]).

\section{B. Stability of the Slow Subsystem}

From (4.5), the eigenvalues of the separated slow subsystem are precisely the eigenvalues of the matrix (the superscript ' $s$ ' indicates slow variables)

$$
A_{0}+B \Gamma=: A^{\mathrm{s}}
$$

if a solution $\Gamma$ of (4.2) exists. Since by (H2) $A_{0}$ is a stable matrix, there exist positive definite matrices $T^{\mathrm{s}}, R^{\mathrm{s}}$ such that

$$
A_{0}^{\prime} R^{\mathrm{s}}+R^{\mathrm{s}} A_{0}=-T^{\mathrm{s}} \text {. }
$$

Proposition 2 now implies that $A^{s}$ is a stability matrix (i.e. the slow modes are stable) if there is a solution $\Gamma$ of (4.2) with $|B \Gamma|<\frac{\left|T^{\mathrm{s}}\right|}{2\left|R^{\mathrm{s}}\right|}$. This will be true if

$$
|\Gamma|<\frac{\left|T^{\mathrm{s}}\right|}{2|B|\left|R^{\mathrm{s}}\right|}
$$

is satisfied, where $\Gamma$ is some solution to Eq. (4.2). 


\section{Stability of the Fast Subsystem}

Recall from Eq. (4.6) that the fast subsystem is given by

$$
\dot{y}=\left(E^{-1}(\epsilon) D+D^{-1} C B-\Gamma(\epsilon) B\right) y=: A^{\mathrm{f}} y,
$$

assuming of course that a solution $\Gamma$ of (4.2) exists. Rather than attempt to apply Proposition 2 to the fast subsystem (4.6), one invokes hypothesis (H3) to study the stability. Proceeding, define the Liapunov function candidate $v(y)$ for $(4.22)$ as

$$
v(y):=y^{\prime} P E(\epsilon) y
$$

where $P$ (now constant) is from Eq. (2.7) of (H3). Evaluating $\dot{v}$ along trajectories of (4.22), one obtains

$$
\begin{aligned}
\dot{v}(y) & =y^{\prime}\left\{D^{\prime} E^{-1}(\epsilon) P E(\epsilon)+P D\right\} y \\
& +y^{\prime}\left\{B^{\prime} C^{\prime}\left(D^{-1}\right)^{\prime} P E(\epsilon)+P E(\epsilon) D^{-1} C B\right\} y \\
& -y^{\prime}\left\{B^{\prime} \Gamma^{\prime} P E(\epsilon)+P E(\epsilon) \Gamma B\right\} y .
\end{aligned}
$$

Since by (H3) $P$ is block diagonal with the same structure as $E(\epsilon)$, it follows that $P$ and $E(\epsilon)$ commute, so that the expression $D^{\prime} E^{-1}(\epsilon) P E(\epsilon)+P D$ in $(4.24)$ can be rewritten as $D^{\prime} P+P D=-Q$ by Eq. (2.9). Now Eq. (2.10) of (H3) and the Schwarz inequality are used to obtain an upper bound on the right side of (4.24). Thus

$$
\begin{aligned}
\dot{v}(y) \leq & -c_{3}|y|^{2}+2|E(\epsilon)||P|\left|D^{-1} C B\right||y|^{2} \\
& +2|E(\epsilon)||B||\Gamma||P||y|^{2} .
\end{aligned}
$$

Therefore to ensure that $\dot{v}(y)<0$ it is sufficient to require that $|E(\epsilon)|$ be bounded from above as follows:

$$
|E(\epsilon)|<\frac{c_{3}}{2|P|\left(\left|D^{-1} C B\right|+|B||\Gamma|\right)},
$$

where $\Gamma$ is any solution of $(4.2)$.

Define $E_{2}(\alpha)$ by

$$
E_{2}(\alpha):=\frac{c_{3}}{2|P|\left(\left|D^{-1} C B\right|+\alpha|B|\right)}
$$

\section{An Upper Bound for Stability}

The foregoing results may now be combined to yield an upper bound $E_{0}$ on $|E(\epsilon)|$ such that for $|E(\epsilon)|<E_{0}, \epsilon \in R_{+}^{M}$, the asymptotic stability of the multiparameter singularly perturbed system (4.1) is certain. By the results of Section NV.2-B, the slow subsystem is well defined and its stability is ensured if there is a solution $\Gamma$ of Eq. (4.2) satisfying the inequality (4.21). By Theorem 9, this will be the case if $|E(\epsilon)| \leq E_{i}^{*}(\alpha)$ where $\alpha$ is any positive number satisfying

$$
\alpha<\frac{\left|T^{s}\right|}{2|B|\left|R^{s}\right|}=: \bar{\alpha}
$$

Similarly, the fast subsystem will be well defined and stable if the last remark holds and if $|E(\epsilon)|<E_{2}(\alpha)$ where $E_{2}(\alpha)$ is given by Eq. (4.27), and where, again, $\alpha$ is any positive scalar satisfying (4.28). By Eq. (4.4) stability of the fast and slow subsystems implies that of the singularly perturbed system (4.1).

For any given $\alpha>0$ satisfying (4.28), clearly an upper bound on $|E(\epsilon)|$ ensuring stability of (4.1) is min $\left(E_{1}^{*}(\alpha), E_{2}(\alpha)\right)$. To optimize the upper bound, one takes the maximum of this quantity over all $\alpha$ satisfying Eq. (4.28). These remarks are summarized in the following theorem, in which the upper bound $E_{0}$ of this paper for time-invariant systems is stated explicitly. 
Theorem 10. Let hypotheses (H1)-(HS) hold, with $P, Q$ constant matrices. Then the null solution of (4.1) is asymptotically stable for all $\epsilon \in R_{+}^{M}$ with $|E(\epsilon)|<E_{0}$ where $E_{0}$ is given by

$$
E_{0}:=\max _{0 \leq \alpha \leq \bar{\alpha}} \min \left\{E_{1}^{*}(\alpha), E_{2}(\alpha)\right\},
$$

and where $E_{1}^{*}(\alpha), E_{2}(\alpha)$ and $\bar{\alpha}$ are given by Eqs. (4.15), (4.27) and (4.28) respectively.

\section{CONCLUSIONS}

The paper has presented a derivation of explicit upper bounds on a weighted norm of the vector of singular perturbation parameters ensuring that the multiparameter singularly perturbed system (1.1) can be decoupled into separate fast and slow subsystems (3.2a,b), and that (1.1) is uniformly asymptotically stable. The results for time-invariant systems apply uniformly as the small parameters approach zero independently, regardless of their relative magnitudes. Thus both the multiple time scales setting $[21,23]$ and the bounded mutual ratios setting [16] are addressed as special cases for time-invariant systems. The upper bound obtained for stability in the timeinvariant case is uniform and so does not display the conservativeness which occurred in Khalil [11] and in the results of the present paper on time-varying systems.

Although the hypothesis (H4) of bounded mutual ratios of the small parameters is valid for a large class of physical systems, it remains an interesting and open question as to the extent to which it can be relaxed for time-varying systems. It is also interesting to consider whether a 'strong D-stability' type assumption can replace hypothesis $(\mathrm{H} 3)$ in the time-varying case. Finally, the relationship between strong D-stability and the conditions for stability of multiple time-scale systems derived by Khalil and Kokotovic [43] should be studied.

\section{ACKNOWLEDGMENT}

The author is grateful to Professor Hassan $\mathrm{K}$. Khalil for his constructive suggestions, and to Professor André L. Tits for several useful discussions.

\section{REFERENCES}

[1] P.V. Kokotovic, "Applications of singular perturbation techniques to control problems," SIAM Review vol. 26, pp. 501-550 (1984).

[2] V.R. Saksena, J. O'Reilly, and P.V. Kokotovic, "Singular perturbations and time-scale methods in control theory: Survey 1976-1983," Automatica vol. 20, pp. 273-294 (1984).

[3] P.V. Kokotovic, R.E. O'Malley, Jr., and P. Sannuti, "Singular perturbations and order reduction in control theory - An overview," Automatica vol. 12, pp. 123-132 (1976).

[4] P.V. Kokotovic, "A Riccati equation for block-diagonalization of ill-conditioned systems," IEEE Trans. Automatic Control vol. AC-20, pp. 812-814 (1975).

[5] J.H. Chow, (Ed.). Tirne-Sicale Modeling of Dynamic Networks with Applications to Power Systems, Springer-Verlag, Berlin (1982).

[6] M.J. Balas, "Stability of distributed parameter systems with finite-dimensional controllercompensators using singular perturbations," J. Math. Anal. Appl. vol. 99, pp. 80-108 (1984).

[7] E.H. Abed, "A new parameter estimate in singular perturbations," Systems and Control Letters vol. 6, pp. 193-198 (1985).

[8] L. Zien, "An upper bound for the singular parameter in a stable, singularly perturbed system," J. Franklin Institute vol. 295. pp. 373-381 (1973).

[9] S.H. Javid, "Uniform asymptotic stability of linear time-varying singularly perturbed systems," J. Franklin Institute vol. 305, pp. 27-37 (1978).

[10] J.H. Chow, "Asymptotic stability of a class of non-linear singularly perturbed systems," $J$. Franklin Institute vol. 305, pp. 275-281 (1978).

[11] H.K. Khalil, "Asymptotic stability of nonlinear multiparameter singularly perturbed 
systems," Automatica vol. 17, pp. 797-804 (1981).

[12] N.R. Sandell, Jr., "Robust stability of systems with application to singular perturbations," Automatica vol. 15, pp. 467-470 (1979).

[13] A. Saberi, Ph.D. Dissertation, Michigan State University, East Lansing (1983).

[14] A. Saberi and H. Khalil, "Quadratic-type Lyapunov functions for singularly perturbed systems," IEEE Trans. Automatic Control vol. AC-29, pp. 542-550 (1984).

[15] K. Khorasani and M.A. Pai, "Asymptotic stability improvements of multiparameter nonlinear singularly perturbed systems," IEEE Trans. Automatic Control vol. AC-30, pp. 802$804(1985)$.

[16] H.K. Khalil and P.V. Kokotovic, "D-Stability and multi-parameter singular perturbation," SIAM J. Control Optim. vol. 17, pp. 56-65 (1979).

[17] H.K. Khalil and P.V. Kokotovic, "Control strategies for decision makers using different models of the same system," IEEE Trans. Automatic Control vol. AC-23, pp. 289-298 (1978).

[18] H.K. Khalil, "Stabilization of multiparameter singularly perturbed systems," IEEE Trans. Automatic Control vol. AC-24, pp. 790-791 (1979).

[19] H.K. Khalil and P.V. Kokotovic, "Decentralized stabilization of systems with slow and fast modes," Large Scale Systems vol. 1, pp. 141-148 (1980).

[20] G.S. Ladde and D.D. Siljak, "Multiparameter singular perturbations of linear systems with multiple time scales," Automatica vol. 19, pp. 385-394 (1983).

[21] A.N. Tikhonov, "Systems of differential equations containing small parameters in the derivatives," Mat. Sb. (in Russian) vol. 31 (73), pp. 575-586 (1952).

[22] W. Wasow, Asymptotic Expansions for Ordinary Differential Equations, Wiley-Interscience, New York (1965)

[23] F. Hoppensteadt, "Properties of solutions of ordinary differential equations with small parameters," Comm. Pure Appl. Math. vol. 24, pp. 807-840 (1971).

[24] R.E. O'Malley, Jr., "Boundary value problems for linear systems of ordinary differential equations involving many small parameters," $J$. Math. Mech. vol. 18, pp. 835-855 (1969).

[25] M. Coderch, A.S. Willsky, S.S. Sastry, and D.A. Castanon, "Hierarchical aggregation of linear systems with multiple time scales," IEEE Trans. Automatic Control vol. AC-28, pp. $1017-1030(1983)$.

[26] R.I. Silva-Madriz, Reduced-Order Modelling of Linear and Nonlinear Multiple Time-Scale Systems, Ph.D. Thesis, Dept. of EECS, Univ. of California, Berkeley (1984).

[27] E.H. Abed, "Multiparameter singular perturbation problems: Iterative expansions and asymptotic stability," Systems and Control Letters vol. 5, pp. 279-282 (1985).

[28] E.H. Abed, “Singularly perturbed Hopf bifurcation," IEEE Trans. Circuits and Systems vol. CAS-32, pp. 1270-1280 (1985).

[29] E.H. Abed, "Strong D-stability," preprint, (1985).

[30] E.H. Abed and A.L. Tits, "On the stability of multiple time-scale systems," Int. J. Control, (to appear).

[31] W.A. Coppel, Dichotomies in Stability Theory, Springer-Verlag, Beriin (1978).

[32] D.D. Siljak, Large Scale Dynamic Systems: Stubility and Structure, North-Holland, New York (1978).

[33] C.R. Johnson, "Sufficient conditions for D-stability," J. Economic Theory vol, 9, pp. 53-62 (1974).

[34] W.A. Coppel, Stabitity and Asymptotic Behavior of Differential Equations, D.C. Heath, Bos$\operatorname{ton}(1965)$

[35] K.W. Chang, "Singular perturbations of a general boundary value problem," SIAM $J$. 
Math. Anal. vol. 3, pp. 520-526 (1972).

[36] K.W. Chang, "Remarks on a certain hypothesis in singular perturbations," Proc. Amer. Math. Soc. vol. 23, pp. 41-45 (1969).

[37] C.J. Harris and J.F. Miles, Stability of Linear Systems, Academic Press, London (1980).

[38] J. Lasalle and S. Lefschetz, Stability by Liapunov's Direct Method, Academic Press, New York (1961).

[39] R.W. Brockett, Finite Dimensional Linear Systems, John Wiley, New York (1970).

[40] M.S. Berger, Nonlinearity and Functional Analysis, Academic Press, New York (1977).

[41] J.K. Hale, Ordinary Differential Equations, 2nd Edition, R.E. Krieger Publishing Co., Malabar, FL (1980).

[42] A.J. Laub, "Numerical linear algebra aspects of control design computations," IEEE Trans. Automatic Control vol. AC-30, pp. 97-108 (1985).

[43] H.K. Khalil and P.V. Kokotovic, "Control of linear systems with multiparameter singular perturbations," Automatica vol. 15, pp. 197-207 (1979). 